\title{
A Case of Symptomatic Inflammatory Tongue Treated with Fucoidan
}

\author{
Shuichi Tsubura ${ }^{1, *}$, Yoshie Waki ${ }^{2}$, Tsutomu Tsubura² \\ ${ }^{1}$ Department of Biochemistry, Nippon Dental University School of Life Dentistry at Niigata, Hamaura-cho, chuo-ku, Niigata, Japan \\ ${ }^{2}$ Tsubura Dental Clinic, Utsunomiya, Tochigi, Japan \\ *Corresponding author: tshu@ucatv.ne.jp
}

Received July 06, 2015; Revised July 13, 2015; Accepted July 16, 2015

\begin{abstract}
Abnormalities of symptomatic inflammatory tongue (SIT) present a diagnostic and therapeutic dilemma for clinicians. In particular, atrophic glossitis or fissured tongue is often linked to underlying semi-pathological conditions such as immunodeficiency, nutritional deficiency, or the effects of pharmacotherapy. The various therapies for this condition include corticosteroid ointment, anesthetic gel, anti-inflammatory drugs, and vitamin B12 supplementation to address the symptoms. However, none of these treatments has been evaluated rigorously. The case of an adult patient in which persistent, painful SIT was successfully treated using a 32\% fucose chewable tablet is presented. In this case, PC- $320^{\mathrm{TM}}$ achieved marked improvements in SIT. Clinical trials are needed to confirm the efficacy and safety of topical PC-320 in treating SIT.
\end{abstract}

Keywords: tongue, fucoidan, inflammation, treatment, tablet

Cite This Article: Shuichi Tsubura, Yoshie Waki, and Tsutomu Tsubura, “A Case of Symptomatic Inflammatory Tongue Treated with Fucoidan.” American Journal of Medical Case Reports, vol. 3, no. 8 (2015): 250-254. doi: 10.12691/ajmcr-3-8-8.

\section{Introduction}

Diagnosis of tongue abnormalities requires examination of tongue morphology and a thorough history, including onset and duration, as well as intra-oral lifestyle habits, such as use of tobacco products or intake of alcoholic beverages. The National Health and Nutrition Examination Survey described the prevalence of tongue lesions as $15.5 \%$ among adults in the United States. The most common abnormal condition of the tongue is geographic tongue, followed by fissured tongue and hairy tongue, as noted by Shulman and others (2004).[1] Symptomatic inflammatory tongue (SIT) often involves atrophic glossitis, fissured tongue, median rhomboid glossitis, or burning tongue. Several other tongue abnormalities are asymptomatic, such as tongue discoloration (white, red, pink or black), hairy-appearing lesions, erythematous ulceration, a line of thickened, adherent patch or plaque, or an isolated pedunculated lesion.

The clinical characteristics of SIT are well-defined, although the precise etiology remains unclear or incomplete, and treatment is non-definitive. The diagnosis, clinical presentation, and severity of SIT are the key factors in determining the selection of treatment. Treatment objectives include controlling pain, suppressing the inflammatory response, and improving the patient's quality of life. Drug delivery represents a major challenge, because topical medications are easily rubbed or rinsed away from the target area through normal oral movements and the flow of saliva. Topical corticosteroid ointment use in patients with SIT is intended to control the inflammatory process associated with the formation of SIT, but side effects may include burning, altered taste, and secondary oral candidiasis. Although some effectiveness of corticosteroid ointment has been shown in treating SIT, the disease course remains unaffected. Furthermore, treatment for a month or more is required to reduce the symptoms.

Fucoidans are a class of sulfated polysaccharides. These fucose-rich polymers were identified in brown algae by Kylin in 1918. [2] Research into fucoidans is progressing into bioactivity and discussion of potential therapeutic applications, which may include antiviral, antibacterial, anticoagulant, and antitumoral activities. [3,4] Despite many studies attempting to determine applications for the bioactivity of fucoidans in medicine and cosmetics, few studies have examined their impacts on oral diseases. A case of painful SIT that proved unresponsive to various medications is reported. This case was successfully treated using topical application of a commercialized oral tablet (PC-320 ${ }^{\mathrm{TM}}$, 32\% fucose chewable tablet; Daiichi-Sangyo, Osaka, Japan). This is the first report describing topical use of PC-320 for SIT.

\section{Case Report (Figure 2A-J)}

A 45-year-old Japanese woman visited our clinic with painful SIT. She explained that her lesions had first developed several years earlier. Treatment with various medications had been attempted, including topical corticosteroid ointment (triamcinolone acetonide) and 
non-steroidal anti-inflammatory drugs, but the lesions did not respond to any of these treatments.

A history of immunosuppressive drug use as medical treatment was elicited. Laboratory investigation identified some risk of anemia or systemic lupus erythematosus on the basis of blood biochemical screening tests. In particular, white blood cell (WBC), hemoglobin ( $\mathrm{Hb})$, and hematocrit (Ht) levels were abnormall (Table 1).

Table 1. WBC, Hb, Ht, AATT, and CRP levels over time

\begin{tabular}{|c|c|c|c|c|c|c|}
\hline & WBC $\left(\times 10^{9} / 1\right)$ & $\mathrm{Hb}(\mathrm{g} / \mathrm{dl})$ & Ht (\%) & APTT & CRP (mg/dl) & Figure \\
\hline Before treatment & 5.30 & 10.5 & 34.4 & 35.9 & 0.10 & $2 \mathrm{~A}$ \\
\hline After 1 week & 3.10 & 9.6 & 32.7 & 37.8 & 0.10 & $2-B$ \\
\hline 2 weeks & 3.80 & 8.8 & 29.8 & 28.3 & 0.10 & $2-C$ \\
\hline 1 month & 4.20 & 9.9 & 33.4 & 28.7 & 0.10 & $2-\mathrm{D}$ \\
\hline 3 months & 6.20 & 11.4 & 36.8 & 44.9 & 0.15 & $2-E$ \\
\hline 6 months & 4.70 & 12.8 & 39.1 & 33.3 & 0.10 & $2-\mathrm{F}$ \\
\hline 12 months & 4.70 & 14.3 & 42.7 & 36.7 & 0.10 & $2-G$ \\
\hline 18 months & 3.30 & 12.9 & 38.7 & 32.3 & 0.10 & $2-\mathrm{H}$ \\
\hline 24 months & 3.80 & 13.4 & 39.3 & 36.9 & 0.10 & 2-I \\
\hline 36 months & 3.75 & 13.2 & 38.7 & 36.5 & 0.10 & 2-J \\
\hline
\end{tabular}

WBC ; White blood cell

$\mathrm{Hb}$; Hemoglobin

Ht ; Hematocrit

APIT ; Activated partial thromboplastin time

CRP ; C reaction proteins.

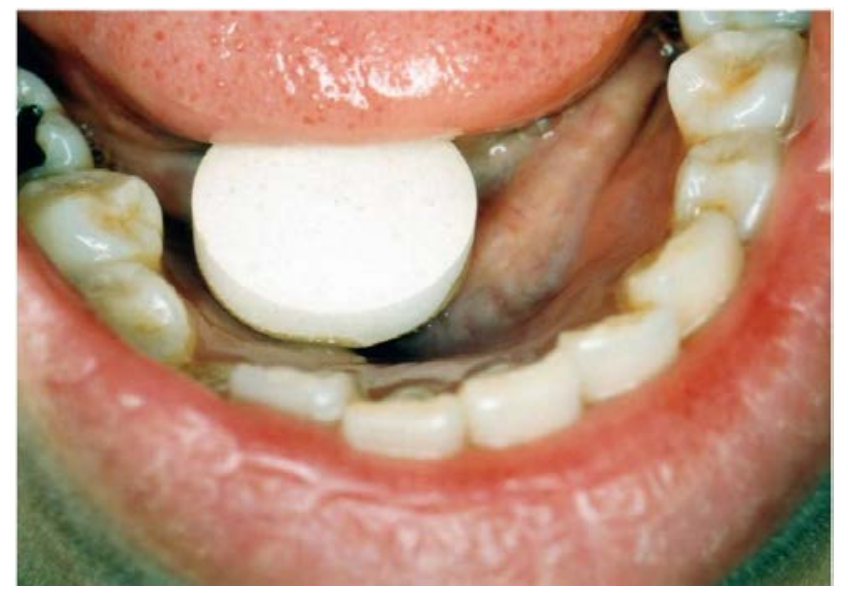

Figure 1. PC- $320^{\mathrm{TM}}$ tablet on the patient's tongue

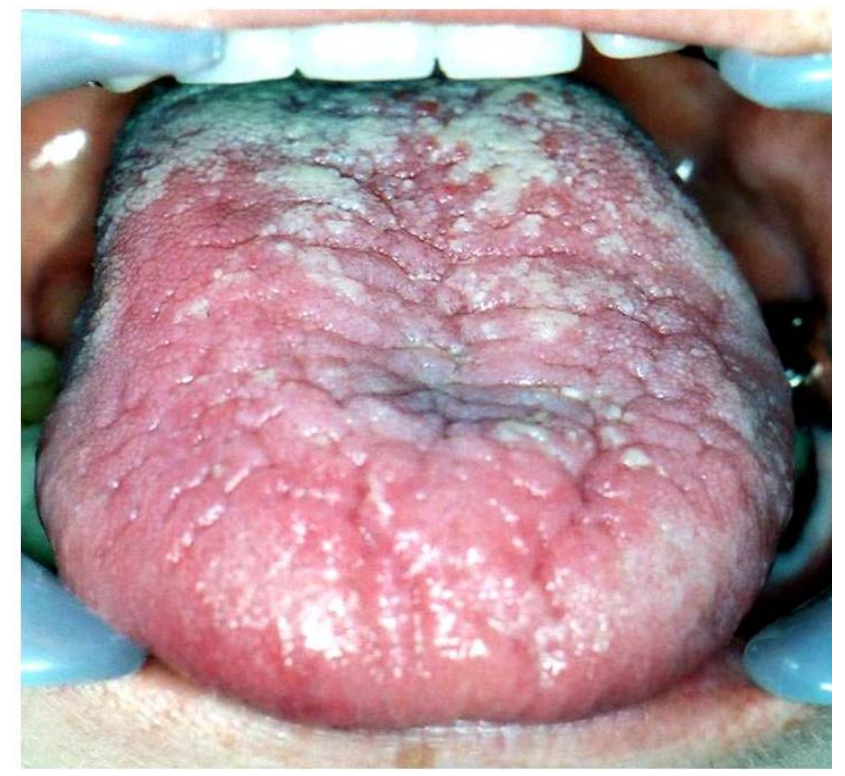

Figure 2. A) Before treatment

Treatment with PC-320 was started, used after meals twice a day, in the morning and evening (Figure 1).
Within 1 week, the condition of the tongue was slightly improved without any side effects and no exacerbation. The patient then consented to further use of PC-320. After 1 month, the patient's clinical condition was dramatically improved, without any complaints from the patient. Follow-up at 3, 6, 12, 18, 24, and 36 months revealed no esthetic or clinical problems, and no systemic activity of PC-320 was identified, as results of blood examinations were unchanged. When the patient first visited us, dermatological and clinical examinations revealed fissured tongue (Figure 2A), in which deep grooves can develop, and also annular white and brown plaques on the middle part of the tongue, malodor, dryness, inflammation, and trapping of food. Treatment with PC-320 twice a day for 3 months showed improvement of FT within 1 month (Figure 2B-D), without any side effects. Biochemical data showed some variability, but the patient's general condition was stabilized (Table 1).

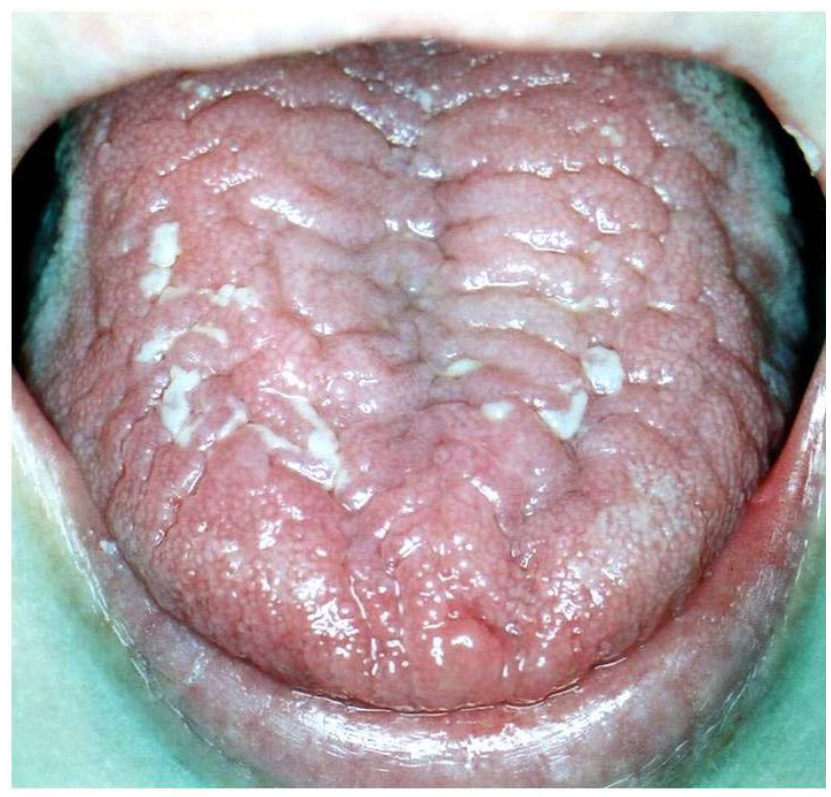

Figure 2. B) After 1 week 


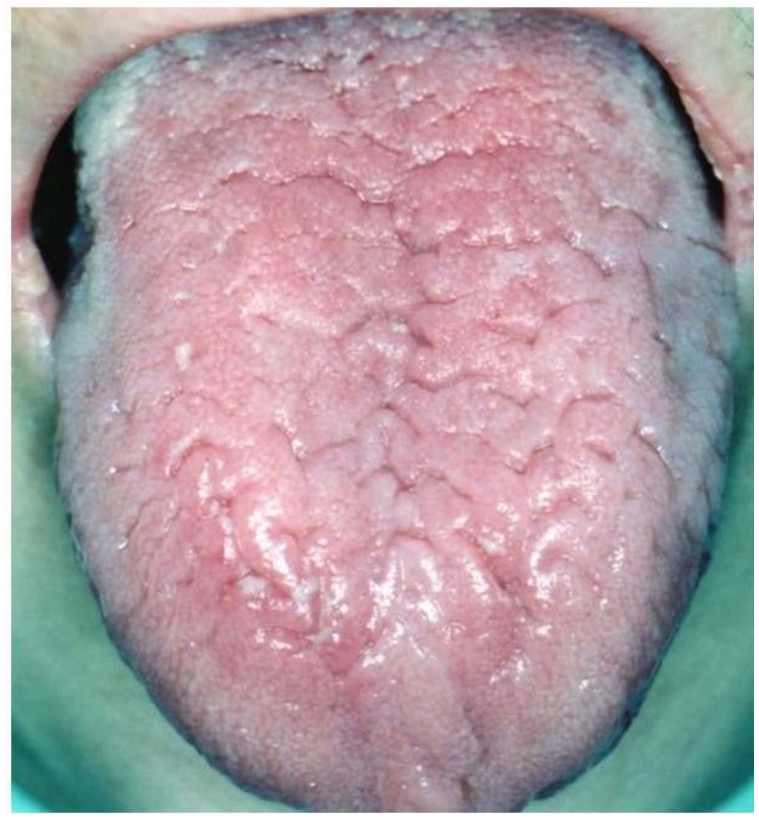

Figure 2. C) After 2 weeks.

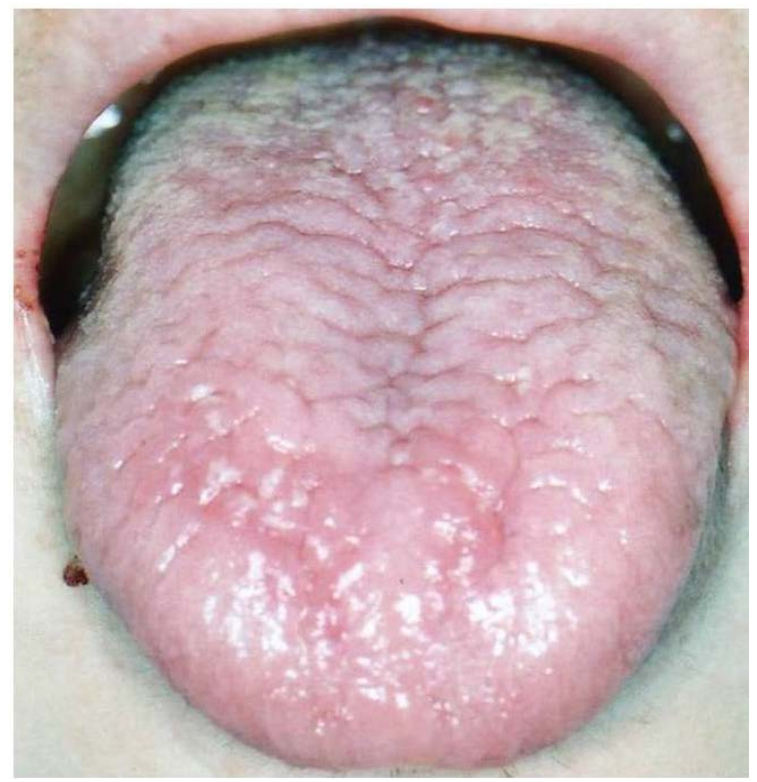

Figure 2. D) After 1 month.

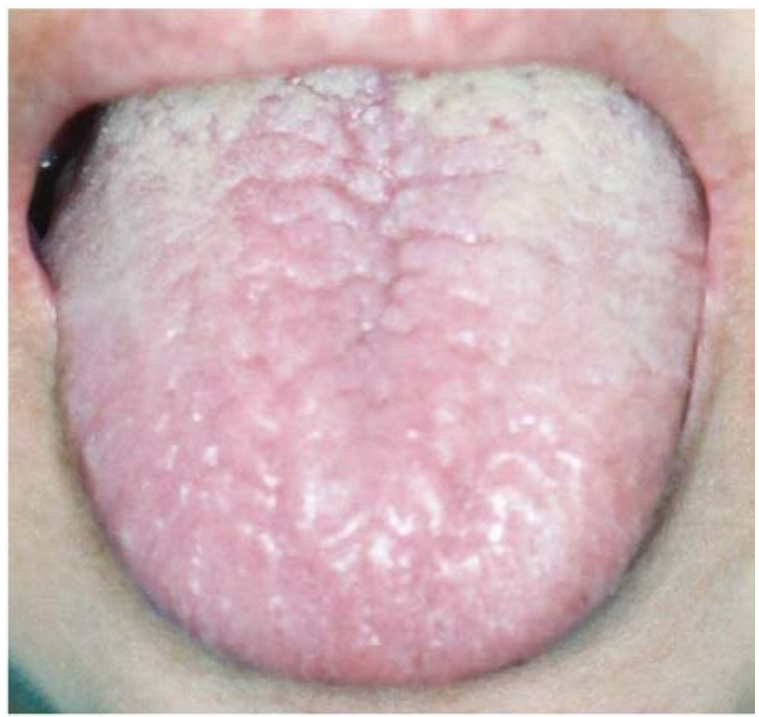

Figure 2. E) After 3 months.

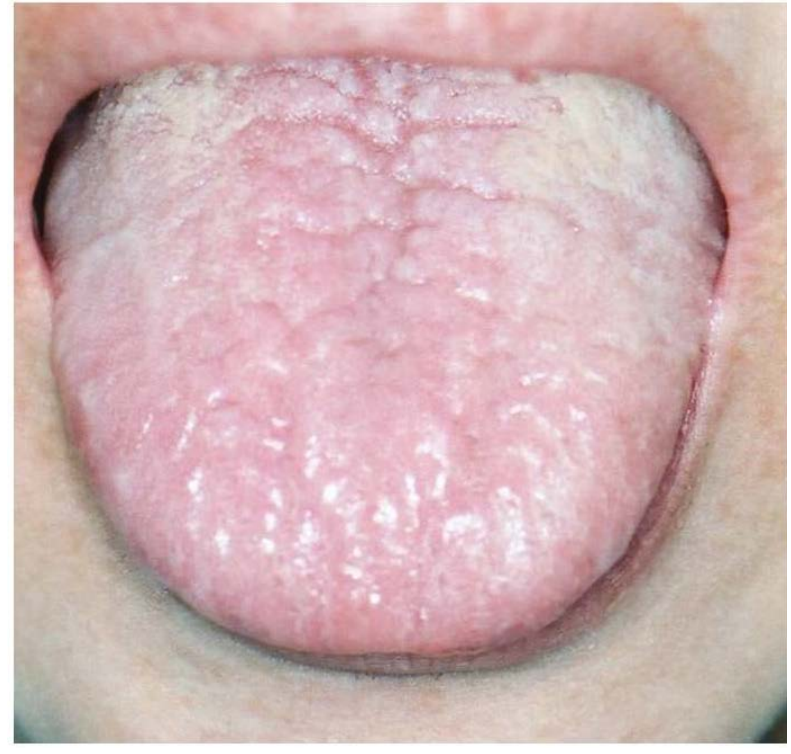

Figure 2. F) After 6 months.

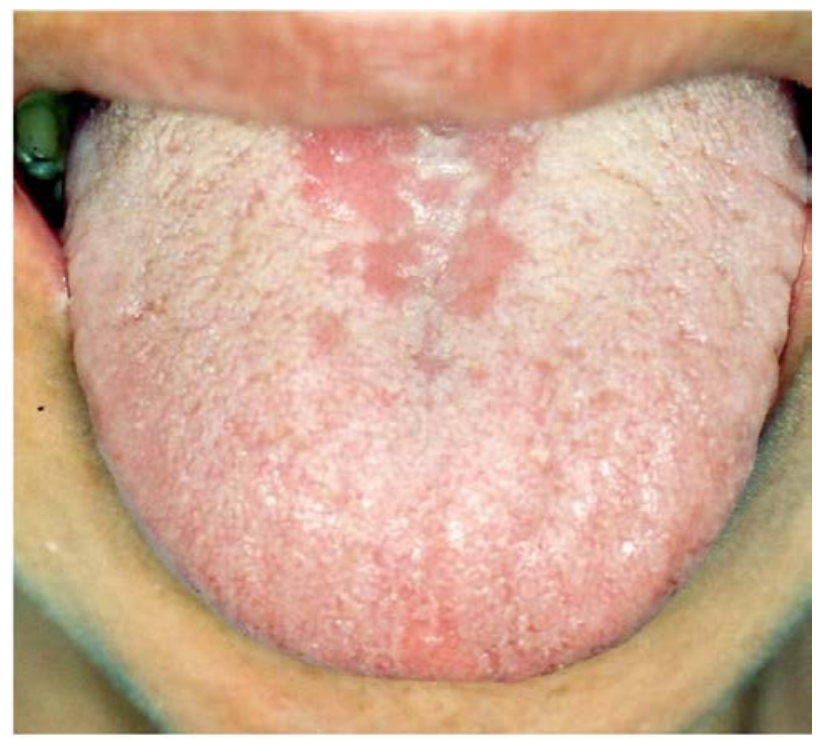

Figure 2. G) After 12 months.

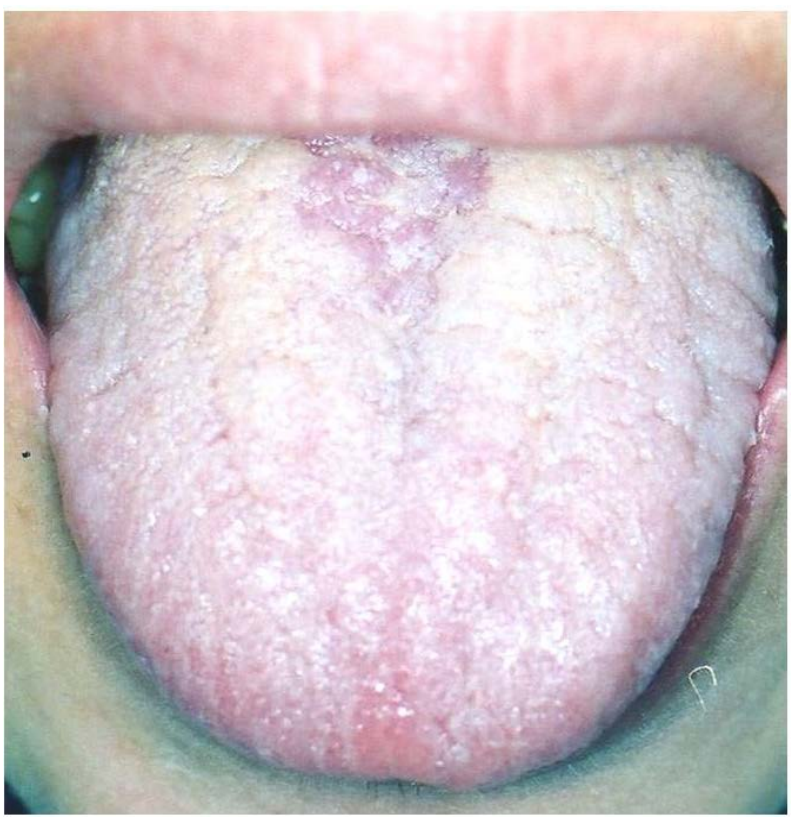

Figure 2. H) After 18 months. 


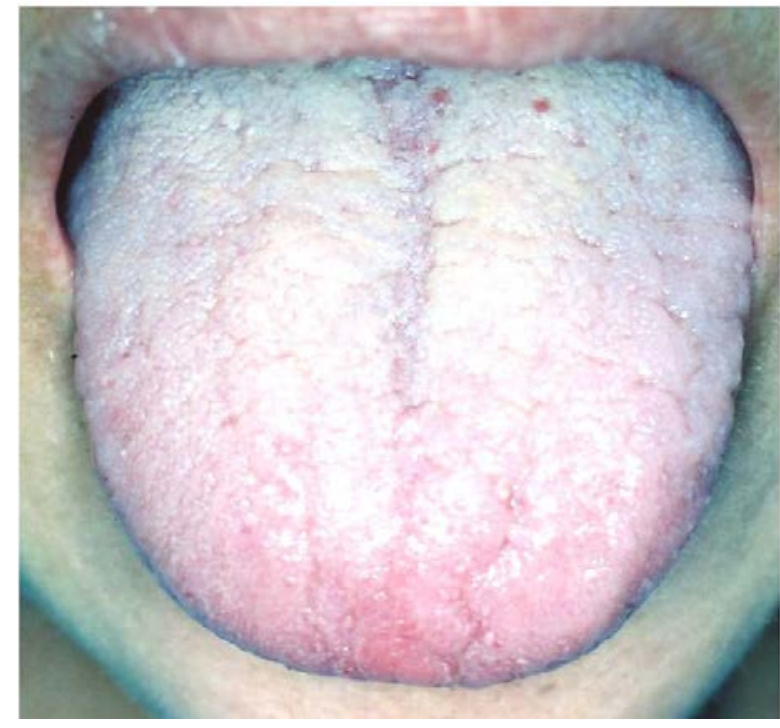

Figure 2. I) After 24 months.

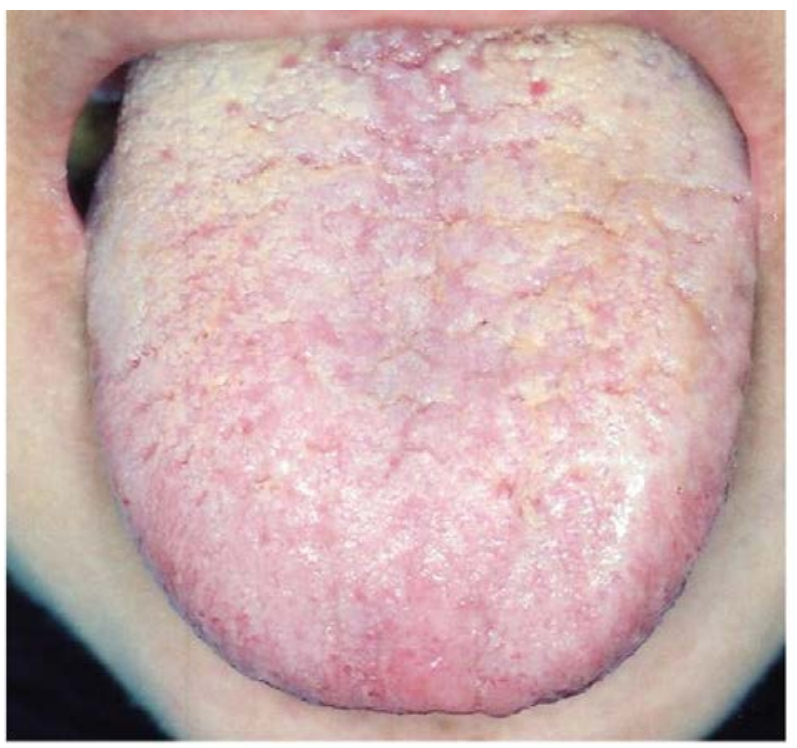

Figure 2. J)After 36 months

\section{Discussion}

SIT is a mucous inflammatory condition of unknown etiology. The typical clinical conditions are atrophic glossitis, fissured tongue, and burning tongue. Other abnormalities of the tongue such as geographic tongue, hairy tongue, and linea alba are usually asymptomatic, and only rarely does significant pain develop and persist; thus, patients with these findings do not usually require treatment. The present patient complained of strong lesional pain and difficulty with food intake for several years. In this case, fissured tongue or burning tongue was considered likely based on clinical circumstantial evidence, although a biopsy was not performed. In general, FT has been associated with Down syndrome, acromegaly, psoriasis, and Sjögren's syndrome. [5] Burning tongue also has an unknown etiology and seems to affect women seven times more often than men. [6] The condition is benign and sometimes resolves spontaneously after many years. Treatment has been attempted using alpha-lipoic acid, clonazepam, cognitive behavior therapy, and treatment of underlying conditions, but none of these approaches has proven particularly effective.

Treatment with fucoidan appears much more effective than various other commercial medications. Study of fucoidan has been increasing in recent years. Siddhanta and Murthy (2001) [7] reported that fucoidan showed antitumoral and anti-inflammatory effects, and Aisa et al. (2005) [8] reported that fucoidan exhibits anti-cancer effects, such as against human lymphoma HS-Sultan cells. With such activities, fucoidan shows high pharmaceutical potential with various possible applications, including use as an anti-viral medication or cancer therapy.

PC-320 contains $32 \%$ fucose in a tablet. The fucose is extracted from Nemacystus decipiens from Tonga and Chile. The molecular weight of this material is under 0.5 $\mathrm{kDa}$. Tablet base components include a maltose-starch stearic acid glyceryl, such as lectin or adenosine triphosphate 2Na, sodium alginate, and others to enhance cell wall stability. The patient is instructed to dab the affected area of the tongue dry, apply the tablet, and allow it to melt on the tongue for $10-15 \mathrm{~min}$, and to avoid eating or drinking for $30 \mathrm{~min}$ after application.

After using PC-320, the present patient experienced no side effects and did not complain of stinging after topical use. The most dramatic effect was the speed of healing. Fucoidan appears to cause both tissue remodeling and repair processes through enzyme-like anti-inflammatory effects. [9] This activity occurs via the inhibition of enzymes including matrix metalloproteinases, hyaluronidases, and elastases [10].

The results of PC-320 therapy in the present case are difficult to interpret scientifically because of the possibility that the observed response may merely reflect the natural course of the disease rather than the effect of the medication. In this case, PC-320 may have been effective in treating the condition, because the previously persistent lesions have not recurred since topical treatment with fucoidan tablets. Further clinical trials are necessary to confirm the efficacy of topical fucoidan for SIT.

\section{Acknowledgments}

The authors wish to thank Dr. Ken Yaegaki for helpful and critical discussions.

\section{Statement of Competing Interests}

The authors have no competing interests.

\section{List of Abbreviations}

\author{
FT, fissured tongue \\ $\mathrm{Hb}$, hemoglobin \\ $\mathrm{Ht}$, hematocrit \\ SIT, symptomatic inflammatory tongue \\ WBC, white blood cell
}

\section{References}

[1] Shulman, J.D., Beach, M.M and Rivera-Hidalgo, F., "The prevalence of oral mucosal lesions in U.S. adults: data from the 
Third National Health and Nutrition Examination Survey, 19881994," J Am Dent Assoc, 135(9). 1279-86. Sep. 2004.

[2] Kylin, H., "Biochemistry of seaweeds," Z Physiol Chem, 101: 236-45. 1918.

[3] Berteau, O. and Mulloy, B., "Sulfated fucans, fresh perspectives: structures, functions, and biological properties of sulfated fucans and an overview of enzymes active toward this class of polysaccharide," Glycobiology,13(6). 29-40. Jun. 2003.

[4] Ribeiro, A.C., Vieira, R.P., Mourao, P.A.S. and Muloy B, "A sulfated alpha-L-fucan from sea cucumber," Carbohydr Res, 255(4):225-40. Mar. 1994.

[5] Byrd, J.A., Bruce, A.J. and Rogers, R.S. III, "Glossitis and other tongue disorders,” Dermatol Clin, 21 (1). 123-34. Jun. 2003.

[6] Drage, L.A. and Rogers, R.S. III, "Burning mouth syndrome," Dermatol Clin, 21(1). 135-45. 2003.
[7] Siddhanta, A.K. and Murthy, A.S.K., "Bioactive polysaccharides from marine brown algae (Phaeophyceae)," J Indian Chem Soc, 78 431-7. 2001.

[8] Aisa, Y., "Fucoidan induces apoptosis of human HS-sultan cells accompanied by activation of caspase-3 and down-regulation of ERK pathways," Am J Hematol, 78(1) .7-14. Jun. 2005.

[9] Senni, K., Gueniche, F., Foucault-Bertaud, A., Igondjo-Tchen, S., Fioretti, F., Colliec-Jouault, S., Durand, P., Guezennec, J., Godeau, G. and Letourneur, D., "Fucoidan a sulfated polysaccharide from brown algae is a potent modulator of connective tissue proteolysis,” Arch Biochem Biophys, 445(1). 56-64. Jan. 2006.

[10] Moon, H.J., Lee, S.H., Ku, M.J., Yu, B.C., Jeon, M.J., Jeong, S.H., Stonik, V.A., Zvyagintseva, T.N., Ermakova, S.P. and Lee, Y.H., "Fucoidan inhibits UVB-induced MMP-1 promoter expression and down regulation of type I procollagen synthesis in human skin fibroblasts,” Eur J Dermatol, 19(2). 129-34. Mar-Apr. 2009. 